\title{
Improved Carrier Frequency Offset Estimation Algorithms for PUCCH Format 1 in LTE-Advanced
}

\author{
Dan Wang, Youbo Feng and Yiming Lin \\ Key Laboratory of Mobile Communication Technology of Chongqing, \\ Chongqing University of Posts and Telecommunications, China \\ youbofeng@163.com
}

\begin{abstract}
Physical Uplink Control Channel (PUCCH) is an important narrow-band channel in LTE-Advanced system, which carries the uplink control information, such as acknowledge/non-acknowledge (ACK/NACK) bits, downlink channel quality indicator (CQI), HARQ-ACK feedback information, and it requires much higher transmission quality than the other channels. With the aid of orthogonal CDMA, multiple users share the same time-frequency resource, the multiuser interference has been the main factor that limits the transmission quality of the control information [1]. In the high-speed mobile scenario, there exists a large Doppler frequency shift, which leads to loss of code orthogonality and also introduces inter-carrier interference (ICI) within a single SCFDMA symbol. Besides, the multiuser interference in high-speed mobile environment is severe. In this paper, the carrier frequency offset estimation algorithms are analyzed, and a double-iteration carrier frequency offset (CFO) estimation algorithm is proved to perform the best for PUCCH Format 1 in high-speed mobile scenario. The estimation process is achieved by coarse estimation and fine estimation, which enlarges the estimation range and improves the estimation accuracy.
\end{abstract}

Keywords: LTE-advanced, PUCCH, carrier frequency offset estimation, doubleiteration

\section{Introduction}

High-speed mobile, causing a great deal of Doppler frequency shift, so that the LTEAdvanced system for broadband communications in high-speed mobile environment faces many challenges. In uplink multiuser situations, Doppler frequency shift, not only will cause inter-carrier interference, also lead to the loss of the codeword orthogonality, resulting in multiuser interference (MUI), hence the need for carrier frequency offset estimation and compensation [2].

At present, carrier frequency offset estimation has been widely studied, however in high-speed mobile environment, the estimation accuracy of traditional carrier frequency offset estimation method is reduced, or estimated range is smaller. Therefore, LTEAdvanced system PUCCH carrier frequency offset estimation in high-speed mobile environment is one of the most urgent problems to be solved. The resource of uplink in LTE-A can be used to estimate carrier frequency offset is the pilot symbols, cyclic prefix, the data symbols, so the carrier frequency offset estimation algorithm can be divided into estimation method based on pilot and based on cyclic prefix [3]. Two kinds of algorithms have their own advantages, estimation method based on pilot for low complexity, easy to implement, but affected by pilot symbol density and interval, the estimation accuracy is low. Estimation method based on cyclic prefix for high complexity, the estimation accuracy will be seriously affected in low SNR and larger delay spread environment.

PUCCH adopts Code Division Multiple Access mechanism [4], users have occupied the same resource in time domain and frequency domain, so it has brought a lot of MUI, 
for every user, there is a lot of noise, and PUCCH occupy few resources. The above problems brought a certain difficulty to the frequency offset estimation. In this paper, introduces several common methods of frequency offset estimation in the frequency domain for PUCCH format1, and on this basis, put forward a frequency offset estimation algorithm suitable for high-speed mobile environment.

\section{PUCCH Signal Model for High-speed Mobile Environment}

Before the analysis of frequency offset estimation algorithm, according to the reference, gives PUCCH signal model of high-speed mobile environment. Let $R_{i}(\mathrm{~m})$ and $R_{i+1}(\mathrm{~m})$ respectively represents $i$ and $i+1$ SC-FDMA symbols in a slot. It can be seen from transmission model of PUCCH:

$$
\begin{aligned}
R_{i}(\mathrm{~m})=\sum_{n=0}^{N-1} r_{i}(\mathrm{n}) \mathrm{e}^{-j 2 \pi n m / N}=\exp \left(\mathrm{j} 2 \pi \varepsilon_{k} \frac{N_{g}+i N_{s}}{N}\right) \mathrm{S}_{k, i} P_{k, i} A_{k, i}(\mathrm{~m}) \alpha_{k}+I_{k, i}(\mathrm{~m})+\mathrm{U}_{k, i}(\mathrm{~m})+W_{i}(\mathrm{~m}) \\
R_{i+1}(\mathrm{~m})=\sum_{n=0}^{N-1} r_{i+1}(\mathrm{n}) \mathrm{e}^{-j 2 \pi n m / N}=\exp \left(\mathrm{j} 2 \pi \varepsilon_{k} \frac{N_{g}+(i+1) N_{s}}{N}\right) \mathrm{S}_{k, i+1} P_{k, i+1} A_{k, i+1}(\mathrm{~m}) \alpha_{k}+I_{k, i+1}(\mathrm{~m})+\mathrm{U}_{k, i+1}(\mathrm{~m})+W_{i+1}(\mathrm{~m})
\end{aligned}
$$

Where $I_{k, i}(\mathrm{~m})$ indicates the $k$-th user inter-carrier interference. $U_{k, i}(\mathrm{~m})$ indicates other users to the $k$-th user interference. $w_{i}(\mathrm{~m})$ indicates the noise. $\alpha_{k}$ indicates sub-carrier signal attenuation coefficient caused by residual frequency offset.

Using orthogonality codeword for multi-user signal despreading, get a single user data, each symbol at user into a sampling point at this time, that is,

$$
\begin{gathered}
B_{k, i}=\sum_{m} R_{i}\left(\mathrm{~m}^{2} \mathrm{~A}_{k, i}{ }^{*}(\mathrm{~m})=12 \exp \left(\mathrm{j} 2 \pi \varepsilon_{k} \frac{N_{g}+\mathrm{iN}_{s}}{N}\right) \mathrm{S}_{k, i} P_{k, i}+I_{k, i}^{\prime}+U_{k, i}^{\prime}+W_{i}^{\prime}\right. \\
B_{k, i+1}=\sum_{m} R_{i+1}\left(\mathrm{~m}^{\prime} \mathrm{A}_{k, i+1}{ }^{*}(\mathrm{~m})=12 \exp \left(\mathrm{j} 2 \pi \varepsilon_{k} \frac{N_{g}+(\mathrm{i}+1) \mathrm{N}_{s}}{N}\right) \mathrm{S}_{k, i+1} P_{k, i+1}+I_{k, i+1}^{\prime}+U_{k, i+1}^{\prime}+W_{i+1}^{\prime}\right.
\end{gathered}
$$

Next, the carrier frequency offset estimation algorithm analysis are carried out on the basis of this model. In order to analysis the performance of carrier frequency offset estimation algorithm, gives the lower bound of the Mean-Square Errors(MSE) Using the frequency offset estimation, such as formula (5).

$$
\operatorname{MSE}(\varepsilon)=\frac{1}{4 \pi^{2}\left(\mathrm{~N}_{s} / \mathrm{N}\right)^{2} N_{p} \cdot n_{a} \cdot N_{\text {block }} \cdot D^{2} \cdot E_{s} / N_{0}}
$$

Where $N_{p}$ represents the total number of taking the average in carrier frequency offset estimation. $n_{a}$ represents the number of receive antennas, $N_{\text {block }}$ indicates the number of carriers in a resource block, D represents the distance between the related operational symbol.

\section{Frequency Offset Estimation Algorithm for PUCCH Format 1}

PUCCH format 1 in a $1 \mathrm{~ms}$ sub-frame, all data symbols send the same information, the pilot symbols transmitted information is fixed. So for the PUCCH format 1, in two consecutive slots, accounting for 6 of pilot symbols of 12 sub carrier and 8 data symbols can be used for frequency offset estimation [5]. This section will introduce respectively based on pilot symbols, based on the data symbols frequency offset estimation algorithm, and based on the pilot symbols and data joint frequency offset estimation algorithm.

Frequency offset in the frequency domain characterized by accumulation of phase rotation, and all sampling points within the same symbol phase rotation is the same. After dispreading in the frequency domain a user remaining 7 sampling points in a slot. We use 
14 sampling points in a sub-frame to PUCCH format $1 / 1 \mathrm{a} / 1 \mathrm{~b}$ frequency offset estimation [6].

\subsection{Frequency Offset Estimation Algorithm based on Pilot Symbols}

Due to 2, 3, 4 symbols for pilot symbols in PUCCH format 1, continuous and send data fixed, so we can use the pilot symbols to estimate the carrier frequency offset. According to the position of pilot symbols, can be divided into adjacent-pilot symbols and intervalpilot symbols frequency offset estimation [7].

\subsubsection{Based on Adjacent-pilot Symbols}

(1) Basic principle

Basic principle of using adjacent-pilot symbols to frequency offset estimation is, the user after the separation in the frequency domain, one symbol for each user within a slot one sampling point left, use only the middle three pilots for carrier frequency offset estimation, every two of three continuous pilot symbol combined to estimate the carrier frequency offset, then the results weighted, as shown in Figure 1.

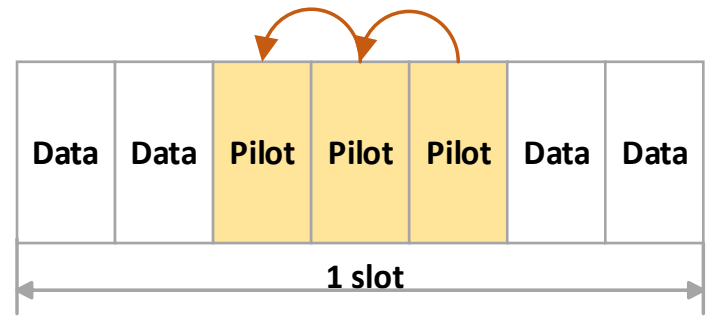

Figure 1. Schematic Diagram of Using Adjacent-pilot Symbols

According to the method as shown in figure 1 for related operation, can get a carrier frequency offset estimation value:

$$
\varepsilon_{k}=\frac{N}{2 \pi N_{s}} \operatorname{angle}\left\{\sum_{m}\left[\frac{B_{k, 3} P_{k}^{*}(3)}{B_{k, 2} P_{k}^{*}(2)}+\frac{B_{k, 4} P_{k}^{*}(4)}{B_{\mathrm{k}, 3} P_{k}^{*}(3)}\right]\right\}
$$

Where $m$ represents a slot number within a sub-frame. By formula (6) can see that this method of carrier frequency offset estimate in the range of $\left[-\mathrm{N} / 2 \mathrm{~N}_{\mathrm{s}}, \mathrm{N} / 2 \mathrm{~N}_{\mathrm{s}}\right]$.

(2) Performance simulation

Simulation parameters are shown in table 1. Among them, the channel environment for AWGN channel with Doppler frequency offset.

Table 1. Simulation Parameters of the Frequency Offset Estimation Algorithm

\begin{tabular}{|c|c|}
\hline Simulation parameter & Value \\
\hline Carrier frequency & $2.3 \mathrm{GHz}$ \\
\hline Bandwidth & $10 \mathrm{MHz}$ \\
\hline CP length & 72 \\
\hline FFT size & 1024 \\
\hline Sub-carrier spacing & $15 \mathrm{KHz}$ \\
\hline Length of a slot & $0.5 \mathrm{~ms}$ \\
\hline Each slot SC-FDMA symbols & 7 \\
\hline Sub-frame interval & Any \\
\hline
\end{tabular}


Consider the case of single user, multiuser, frequency offset estimation using adjacentpilot symbols MSE performance curve, as shown in Figure 2.

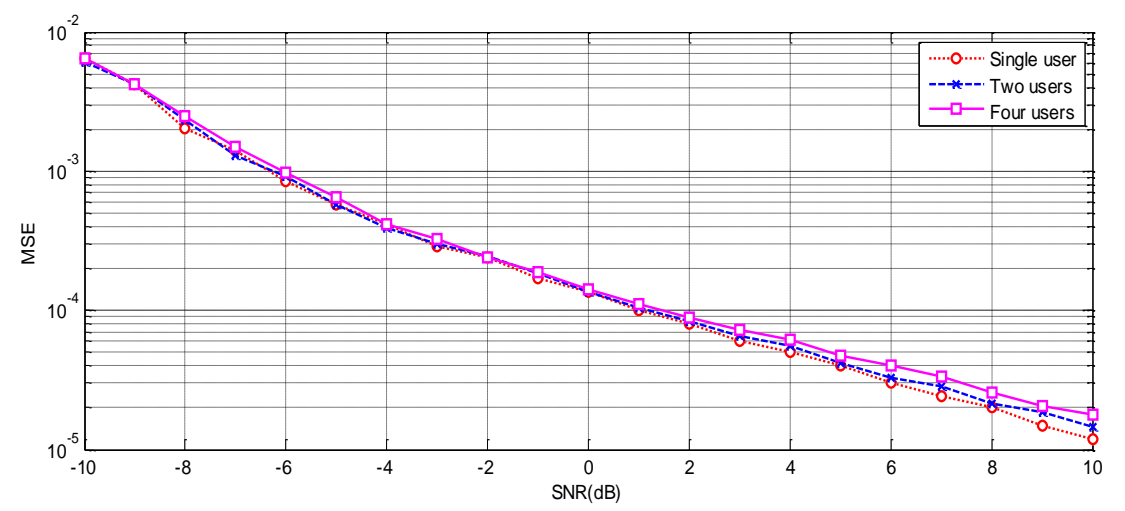

Figure 2. MSE Curves Estimated by the Adjacent-pilot Symbols

We can see from figure 2, the frequency offset estimation using adjacent-pilot symbol, with the increase of the SNR, carrier frequency offset estimation of MSE showed a decreasing trend, and in the $-6 \mathrm{~dB}$, MSE down to $10^{-3}$. Because of the influence of the interference between users, at high SNR, carrier frequency offset estimation performance with slightly decreased as the number of user increases.

\subsubsection{Based on Interval-pilot Symbols}

(1) Basic principle

As the PUCCH format 1 within a slot of three continuous pilot, it can be considered using a symbol interval of pilot for frequency offset estimation. Basic principles of this method is that, after user separation in the frequency domain, a symbol in one slot for each user only one sampling point, using the first and third pilot symbols for carrier frequency offset estimation and its schematic is shown in Figure 3 [8].

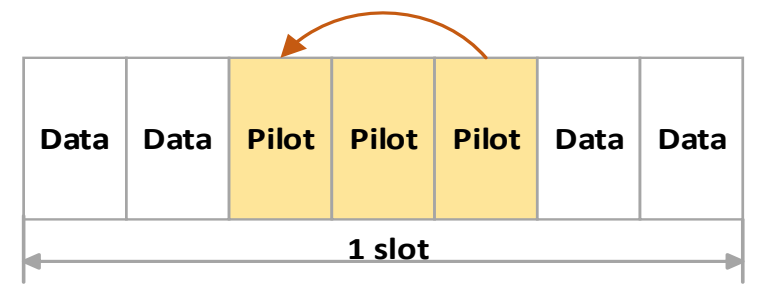

Figure 3. Schematic Diagram of Using Interval-pilot Symbols

According to the method as shown in figure 3 for related operation, can get a carrier frequency offset estimation value:

$$
\varepsilon_{k}=\frac{N}{4 \pi N_{s}} \operatorname{angle}\left\{\sum_{m} \frac{B_{k, 4} P_{k}^{*}(4)}{B_{k, 2} P_{k}^{*}(2)}\right\}
$$

Where $m$ represents a slot number within a sub-frame. By formula (7) can see that this method of carrier frequency offset estimate in the range of $\left[-\mathrm{N} / 4 \mathrm{~N}_{\mathrm{s}}, \mathrm{N} / 4 \mathrm{~N}_{\mathrm{s}}\right]$, which is half of the range estimated by the adjacent-pilot symbols. 


\section{(2) Performance Simulation}

Using interval-pilot to frequency offset estimation of PUCCH format 1, simulation parameters are shown in table 1. Consider the case of single-user, multiuser. Frequency offset estimation using interval-pilot symbols MSE curve, as shown in Figure 4.

We see from Figure 4, the frequency offset estimation using interval-pilot symbol, with the increase of the SNR, carrier frequency offset estimation of MSE showed a decreasing trend, and in the $-7.5 \mathrm{~dB}$, MSE down to $10^{-3}$. Because of the influence of the interference between users, at high SNR, carrier frequency offset estimation performance with slightly decreased as the number of users increases, but the smaller gap between performance.

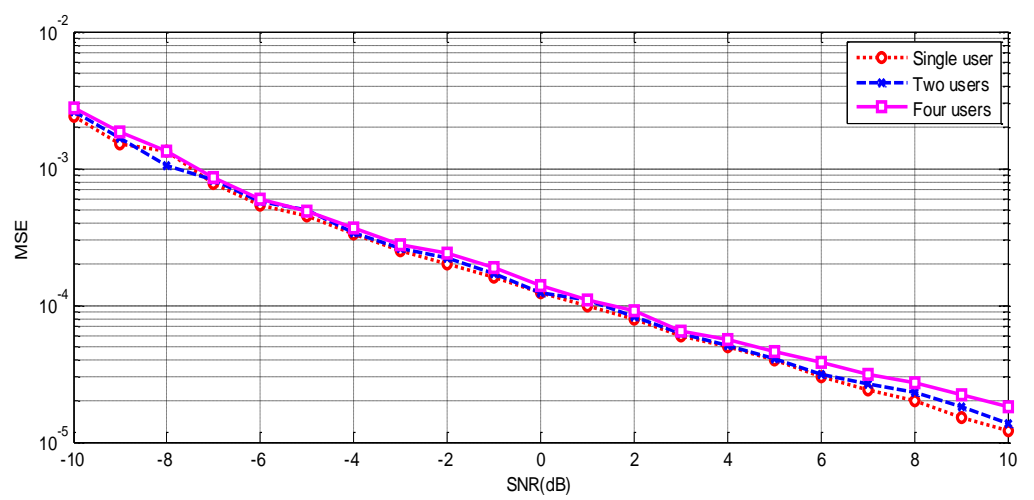

Figure 4. MSE Curves Estimated by the Interval-pilot Symbols

\subsection{Frequency Offset Estimation Algorithm based on Data Symbols}

As four symbols $(0,1,5,6)$ transmit the same data in PUCCH format 1 , so we can use the data symbol to estimate the carrier frequency offset. Depending on where the data symbols, can use adjacent-data symbol and interval-data symbols to estimate the carrier frequency offset [9].

\subsubsection{Based on Adjacent-data Symbols}

\section{(1) Basic principle}

Basic principle of using adjacent-data symbols to frequency offset estimation is, after user separation in the frequency domain, one symbol for each user within a slot one sampling point left. Respectively, use the symbols 0 and 1, 5 and 6 for carrier frequency offset estimation, then the two results weighted, as shown in Figure 5.

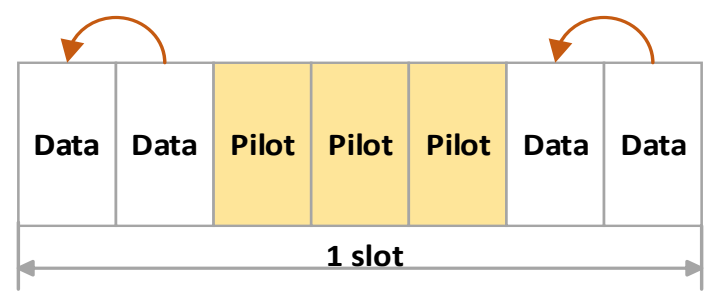

Figure 5. Schematic Diagram of Using Adjacent-data Symbols

According to the method as shown in figure 5 for related operation, can get a carrier frequency offset estimation value:

$$
\varepsilon_{k}=\frac{N}{2 \pi N_{s}} \operatorname{angle}\left\{\sum_{m}\left[\frac{B_{k, 1} P_{k}^{*}(1)}{B_{k, 0} P_{k}^{*}(0)}+\frac{B_{k, 6} P_{k}^{*}(6)}{B_{\mathrm{k}, 5} P_{k}^{*}(5)}\right]\right\}
$$


Where $m$ represents a slot number within a sub-frame. By formula (8) can see that this method of carrier frequency offset estimate in the range of $\left[-\mathrm{N} / 2 \mathrm{~N}_{\mathrm{s}}, \mathrm{N} / 2 \mathrm{~N}_{\mathrm{s}}\right]$.

(2) Performance simulation

Using adjacent-data symbols to frequency offset estimation of PUCCH format 1, simulation parameters are shown in table 1. Consider the case of single-user, multiuser. Frequency offset estimation using adjacent-data symbols MSE curve, as shown in Figure 6.

We can see from Figure 6, the frequency offset estimation using adjacent-data symbol, with the increase of the SNR, carrier frequency offset estimation of MSE showed a decreasing trend, and in the $-5 \mathrm{~dB}, \mathrm{MSE}$ down to $10^{-3}$. Estimate accuracy worse than when using pilot to estimate, and the number of users to estimate at this time has a greater influence on the performance, with the increase of users, interference between user increases, estimation error increases.

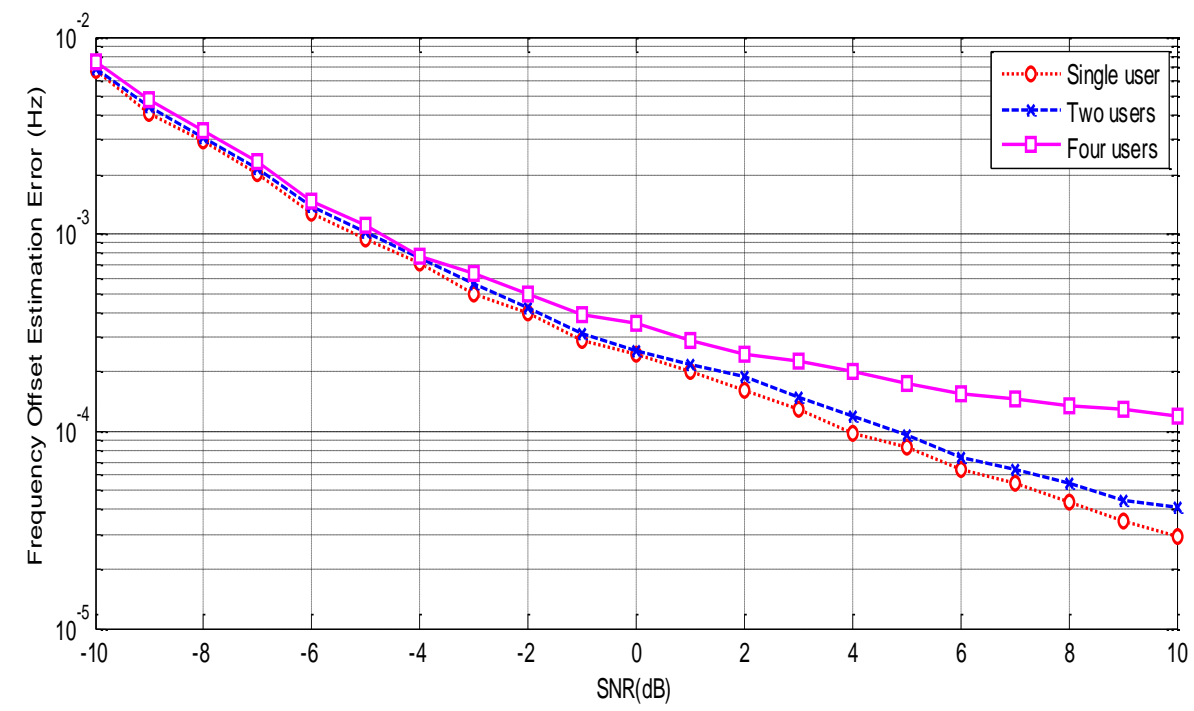

Figure 6. MSE Curves Estimated by the Adjacent-data Symbols

\subsubsection{Based on Interval-data Symbols}

(1) Basic principle

Basic principle of using interval of five data symbols to frequency offset estimation is, after user separation in the frequency domain, one symbol for each user within a slot one sampling point left. Respectively, use the symbols 0 and 5, 1 and 6 for carrier frequency offset estimation, as shown in Figure 7.

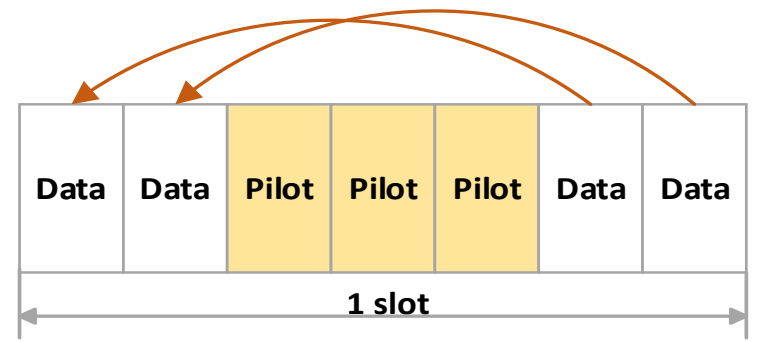

Figure 7. Schematic Diagram of using Interval of Five Data Symbols

According to the method as shown in figure 7 for related operation, can get a carrier frequency offset estimation value: 


$$
\varepsilon_{k}=\frac{N}{10 \pi N_{s}} \operatorname{angle}\left\{\sum_{m}\left[\frac{B_{k, 5} P_{k}^{*}(5)}{B_{k, 0} P_{k}^{*}(0)}+\frac{B_{k, 6} P_{k}^{*}(6)}{B_{\mathrm{k}, 1} P_{k}^{*}(1)}\right]\right\}
$$

Where $m$ represents a slot number within a sub-frame. By formula (9) can see that this method of carrier frequency offset estimate in the range of $\left[-\mathrm{N} / 10 \mathrm{~N}_{\mathrm{s}}, \mathrm{N} / 10 \mathrm{~N}_{\mathrm{s}}\right]$.

\section{(2) Performance simulation}

Using interval of five data symbols to frequency offset estimation of PUCCH format 1, simulation parameters are shown in table 1 . Consider the case of single-user, multiuser, frequency offset estimation using interval of five data symbols MSE curve, as shown in Figure 8 .

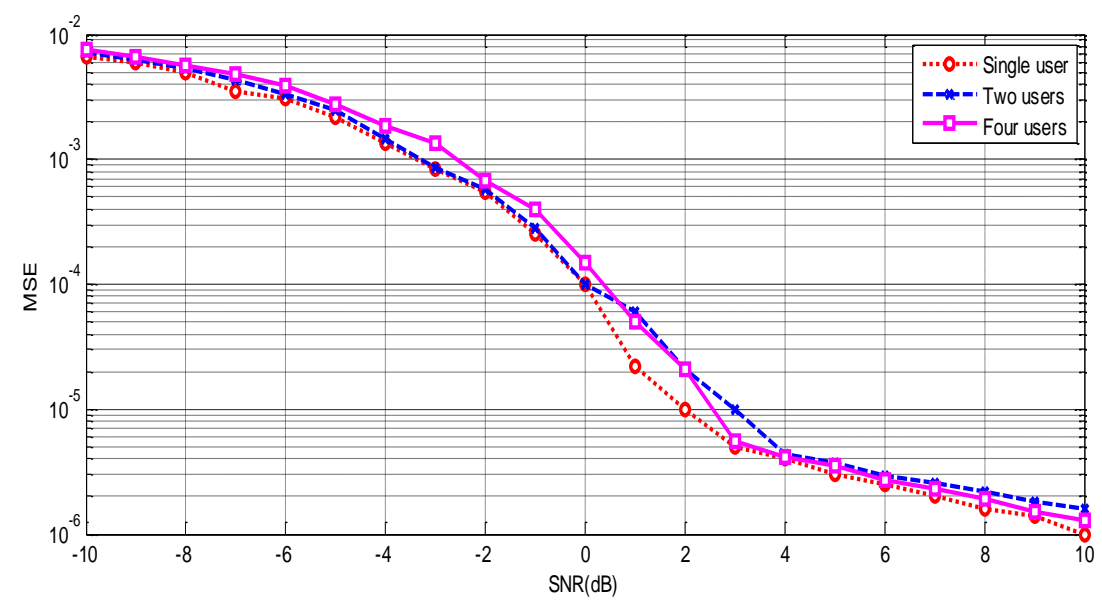

Figure 8. MSE Curves Estimated by the Interval-data Symbols

We can see from Figure 8, the frequency offset estimation using interval-data symbols, with the increase of the SNR, carrier frequency offset estimation of MSE showed a decreasing trend, and in the $-3 \mathrm{~dB}$, MSE down to $10^{-3}$. In addition, this method is poor estimation performance at low SNR, lower estimate accuracy. At high SNR, the performance is better, the estimation accuracy is improved obviously. With the increase of users, little change in estimation accuracy.

\subsection{Joint Frequency Offset Estimation Algorithm based on Pilot and Data Symbols}

The front respectively discuss a method to estimate the carrier frequency offset using the pilot symbols and data symbols, now joint frequency offset estimation algorithm based on pilot and data symbols is discussed.

\section{(1) Basic principle}

Basic principle of joint frequency offset estimation algorithm based on pilot and data symbols is, after user separation in the frequency domain, one symbol for each user within a slot one sampling point left, difference calculation using the same type (data symbol or pilot symbol) of the adjacent sample point, then the results weighted, as shown in Figure 9. 


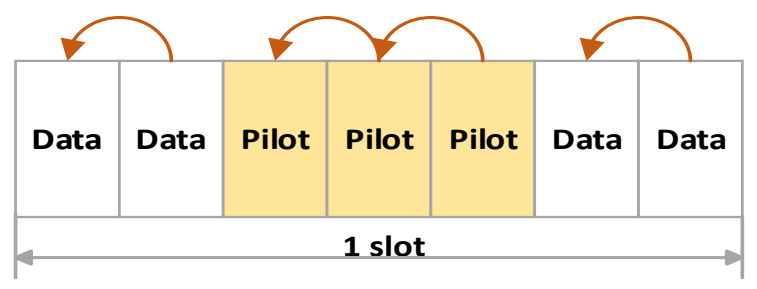

\section{Figure 9. Schematic Diagram of Joint Frequency Offset Estimation Algorithm}

According to the method as shown in figure 9 for related operation, can get a carrier frequency offset estimation value:

$$
\varepsilon_{k}=\frac{N}{2 \pi N_{s}} \text { angle }\left\{\sum_{m}\left[\frac{B_{k, 1} P_{k}^{*}(1)}{B_{k, 0} P_{k}^{*}(0)}+\frac{B_{k, 3} P_{k}^{*}(3)}{B_{k, 2} P_{k}^{*}(2)}+\frac{B_{k, 4} P_{k}^{*}(4)}{B_{\mathrm{k}, 3} P_{k}^{*}(3)}+\frac{B_{k, 6} P_{k}^{*}(6)}{B_{k, 5} P_{k}^{*}(5)}\right]\right\}
$$

Where $m$ represents a slot number within a sub-frame. By formula (10) can see that this method of carrier frequency offset estimate in the range of $\left[-\mathrm{N} / 2 \mathrm{~N}_{\mathrm{s}}, \mathrm{N} / 2 \mathrm{~N}_{\mathrm{s}}\right]$.

\section{(2) Performance Simulation}

Using adjacent-data symbols and adjacent-pilot symbols to frequency offset estimation of PUCCH format 1, simulation parameters are shown in table 1. Consider the case of single-user, multiuser. Joint frequency offset estimation based on pilot and data symbols MSE performance curve, as shown in Figure 10.

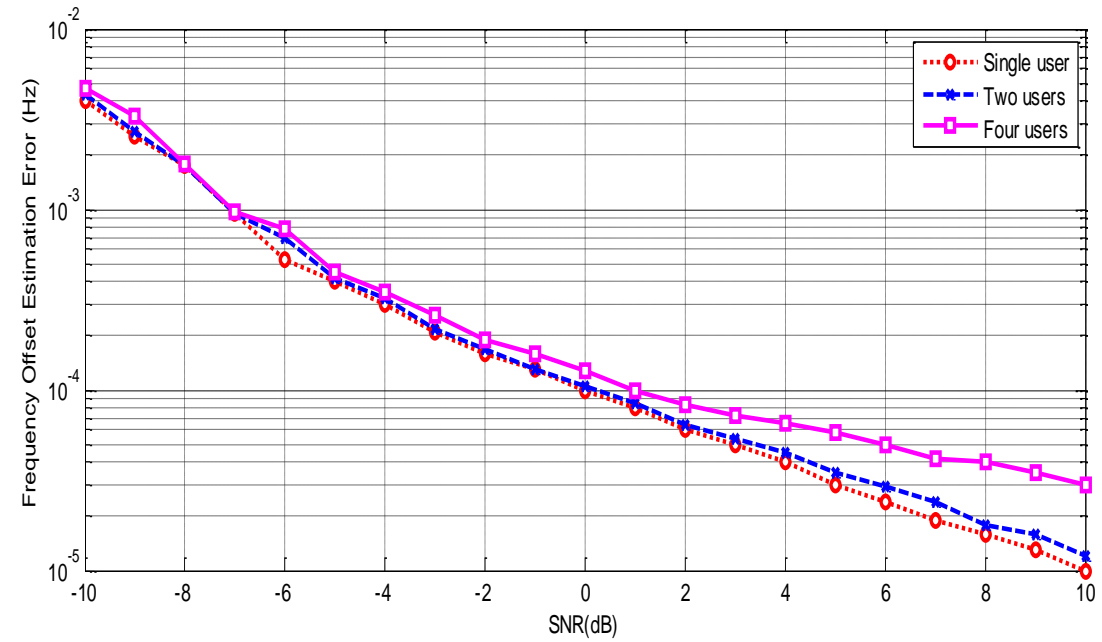

\section{Figure 10. MSE Curves Joint Estimated by Adjacent-data Symbols and Adjacent-pilot Symbols}

We can see from Figure 10, Joint frequency offset estimation based on pilot and data symbols, with the increase of the SNR, carrier frequency offset estimation of MSE showed a decreasing trend, and in the $-7 \mathrm{~dB}$, MSE down to $10^{-3}$. With the increase in the number of users, reduce the accuracy of estimation, and when SNR is greater than $4 \mathrm{~dB}$, the estimation error of four users was higher than that of the single user and two user.

\section{Improved Carrier Frequency Offset Estimation Algorithms}

In the high-speed mobile scenario, there exists a large Doppler frequency shift, which leads to loss of code orthogonality and also introduces ICI within a single SC-FDMA symbol. So carrier frequency offset is very large in the high-speed mobile scenario, if 
using data and pilot symbols to estimate directly, the estimation accuracy is low, for this reason, a double-iteration carrier frequency offset (CFO) estimation algorithm is proved to perform the best for PUCCH Format 1 in high-speed mobile scenario [10].

\section{(1) Basic principle}

Basic principle of double-iteration carrier frequency offset estimation algorithm is, firstly, coarse estimation for CFO using pilot symbols, and to compensate for signal. Then the data symbols are used for fine estimation, to compensate the signal again. By coarse estimation and fine estimation that phase ambiguity can be avoided, and reduce the impact of noise [11].

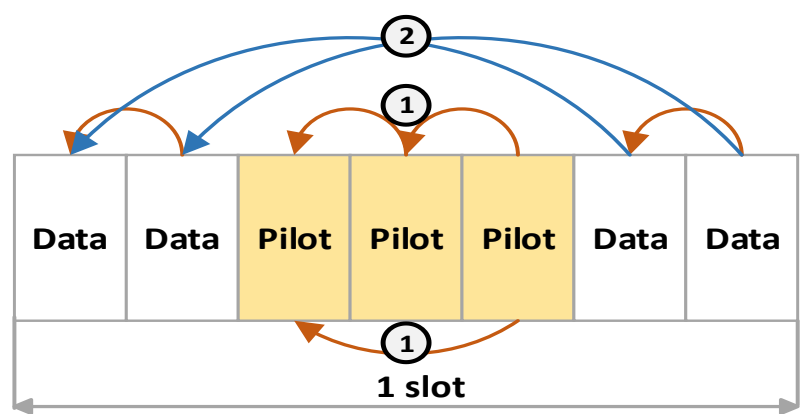

\section{Figure 11. Schematic Diagram of Using Double-iteration}

As shown in Figure 11, Specific procedures are as follows: First of all, using adjacent-pilot symbols and adjacent-data symbols, or the interval-pilot symbols to coarse estimate for CFO. Set frequency offset coarse estimation value for $\varepsilon_{1}$, then compensate the coarse estimation value $\alpha$ times; Then for compensated frequency offset signal, use interval of five data symbols to frequency offset fine estimation, get the value of the carrier frequency offset estimation for $\varepsilon_{2}$. Finally compensates for the this signal;

According to the given earlier PUCCH signal model of high-speed mobile environment, we can get the following formula, the coarse estimation formula (11) of interval-pilot symbols, and fine estimation formula (12) of interval-data symbols, the final frequency offset estimation value can be get by formula (13).

$$
\begin{gathered}
\varepsilon_{k, \text { coarse }}=\frac{N}{4 \pi N_{s}} \operatorname{angle}\left\{\sum_{m}\left[\frac{B_{k, 4} P_{k}^{*}(4)}{B_{k, 2} P_{k}^{*}(2)}\right]\right\} \\
\varepsilon_{k, \text { fine }}=\frac{N}{10 \pi N_{s}} \operatorname{angle}\left\{\sum_{m}\left[\frac{B_{k, 5} P_{k}^{*}(5)}{B_{k, 0} P_{k}^{*}(0)}+\frac{B_{k, 6} P_{k}^{*}(6)}{B_{k, 1} P_{k}^{*}(1)}\right]\right\} \\
\varepsilon_{k}=\alpha * \varepsilon_{k, \text { coarse }}+\varepsilon_{k, \text { fine }}
\end{gathered}
$$

\section{(2) Performance Simulation}

Using double-iteration to frequency offset estimation of PUCCH format 1, simulation parameters are shown in Table 1. Consider the case of a single user, multiuser and different values of the compensation parameter, double-iteration carrier frequency offset estimation algorithm MSE performance curve, as shown in Figure 12 and 13. 


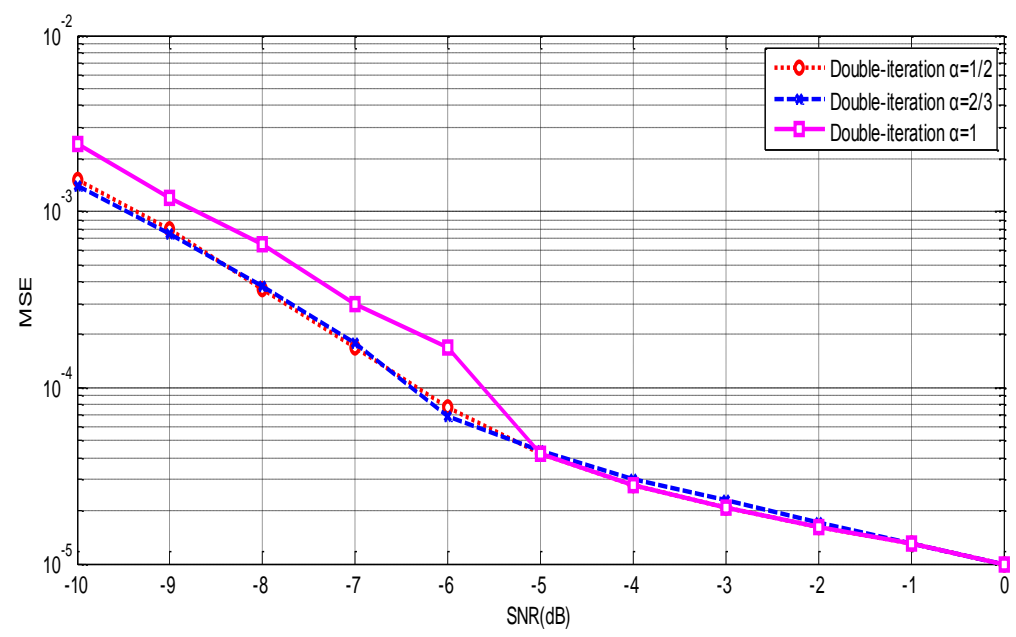

Figure 12. MSE Curves Estimated by Double-iteration in Different $\alpha$ Value

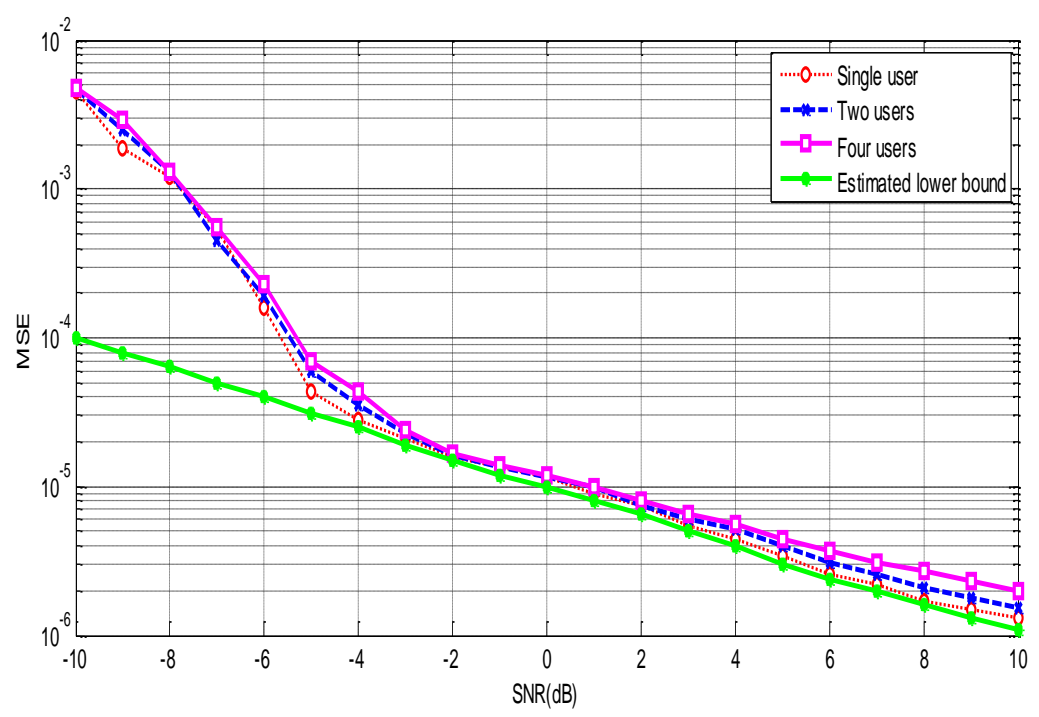

Figure 13. MSE Curves Estimated by Double-iteration in Different Number of Users

Figure 12 shows the case of single user using double-iteration carrier frequency offset estimation algorithm, MSE curves under different value of $\alpha$. It can be seen from figure 12 , in the low SNR, when $\alpha=2 / 3$ and $\alpha=1 / 2$, performance of the frequency offset compensation is better than that of other values. When the SNR greater than $-5 \mathrm{~dB}$, full and partial compensation consistent performance. Partial compensation for performance is better, because in lower SNR, the coarse frequency offset in the first estimate relative to the actual deviation may appear bigger error, so if the completely compensate, the residual frequency offset is large still, then the second estimation of frequency offset must be greater errors(due to limited the range of the second estimate), So for the first estimate, using partial compensation can reduce the impact of the error of the first estimate on the second estimate. Therefore, in the low SNR using partial compensation performance is better.

Figure 13 shows the MSE curves using double-iteration to frequency offset estimation of PUCCH format 1. We can see from Figure 13, double-iteration frequency offset estimation algorithm, with the increase of the SNR, carrier frequency offset estimation of MSE showed a decreasing trend, and in the $-8 \mathrm{~dB}$, MSE down to $10^{-3}$. With the increase in 
the number of users, reduce the accuracy of estimation, when the SNR is greater than $4 \mathrm{~dB}$, estimated MSE is very close to the lower bound of estimation, estimate performance is better, and the algorithm is less affected by the interference between users.

\section{Performance Comparison of Various Algorithms}

Next, frequency offset estimation algorithm performance of all previously described will be compared, simulation parameters are shown in table 1. Consider the case of a single user, multiuser. MSE performance curve of each estimation algorithm shown in Figure 14-16.

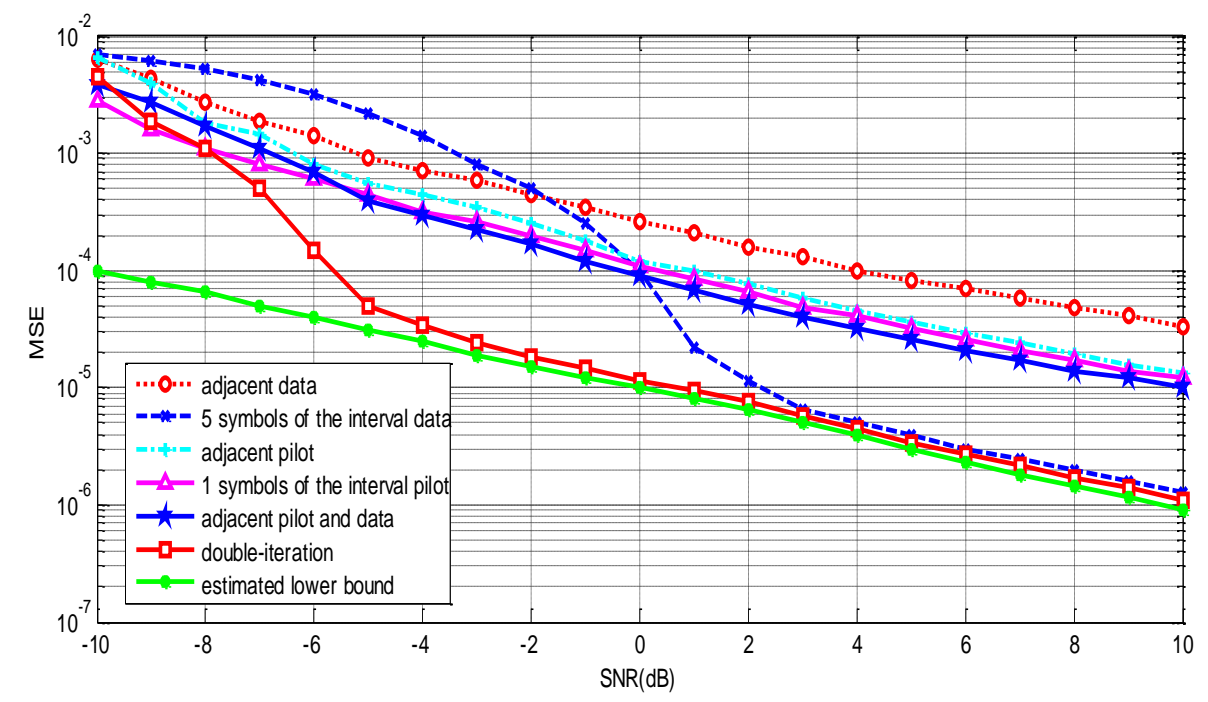

Figure 14. MSE Curves of Each Estimation Algorithm under Single User

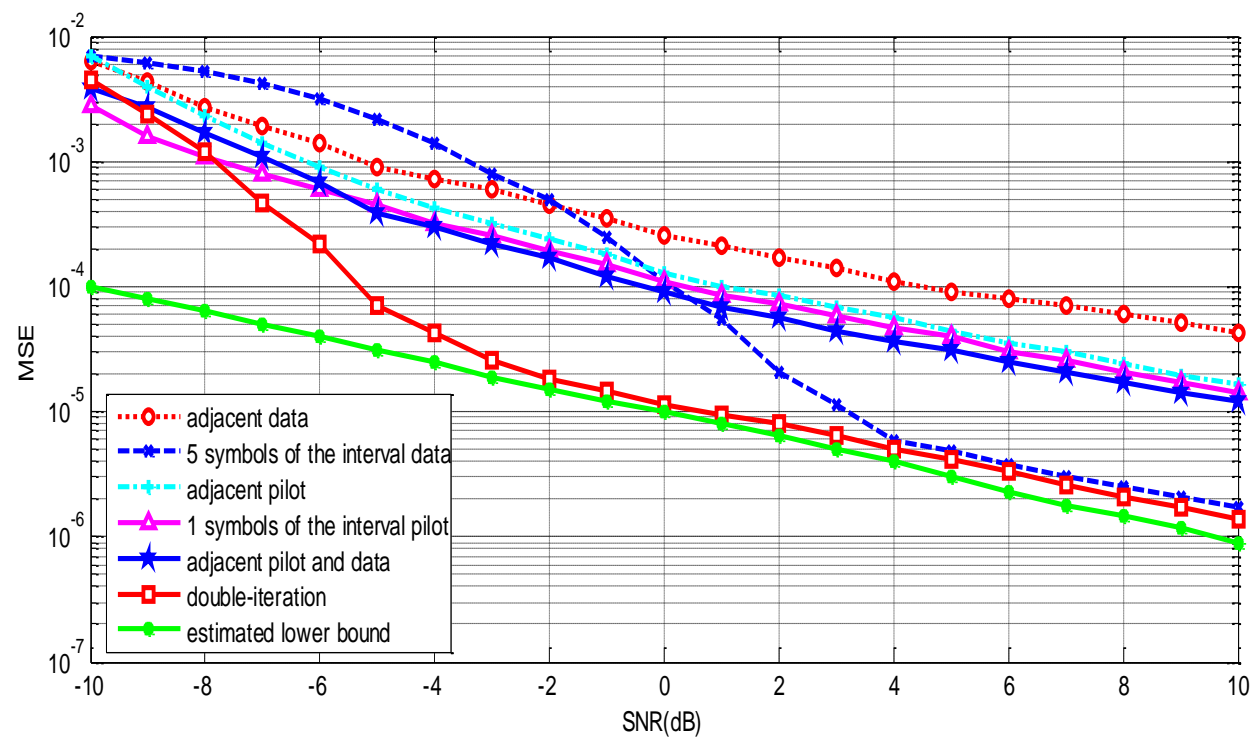

Figure 15. MSE Curves of Each Estimation Algorithm under Two Users 


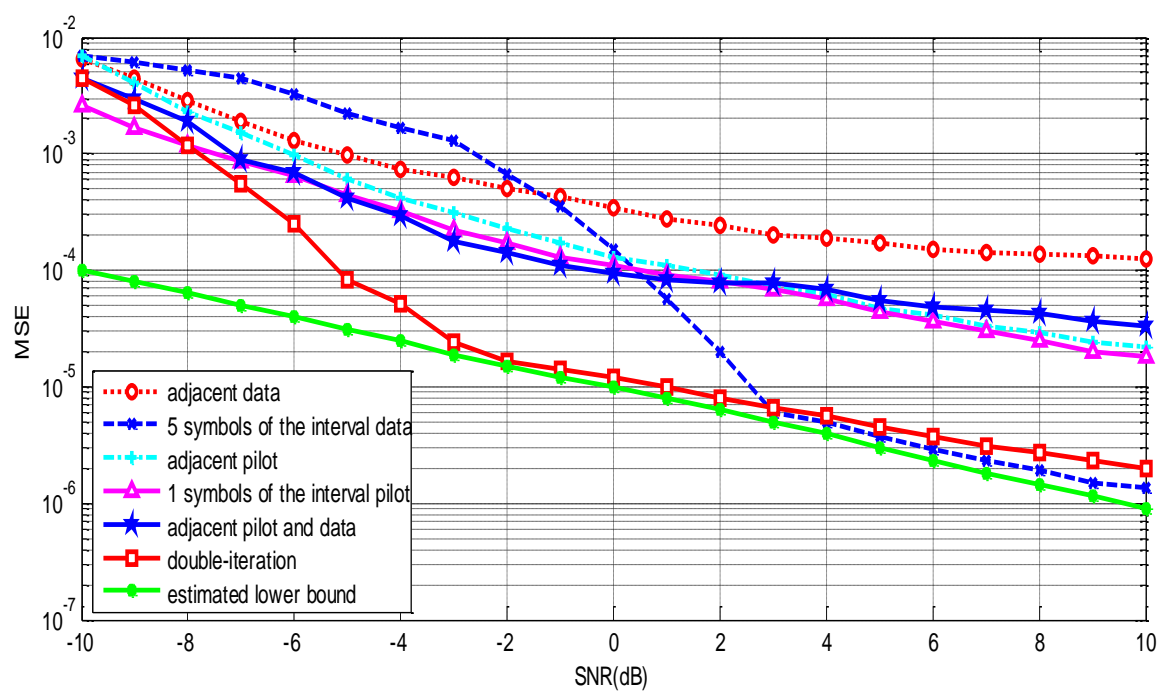

\section{Figure 16. MSE Curves of Each Estimation Algorithm under Four Users}

Figure 14 is single user performance comparison of different algorithm, can be seen from the figure, performance of the double-iteration algorithm is better than other algorithms, and when SNR higher than $-4 \mathrm{~dB}$, estimated MSE is very close to the lower bound of estimation, when the SNR is higher than $3 \mathrm{~dB}$, frequency offset estimation algorithm using interval of five data symbols, estimation accuracy is higher, which consistent with performance of the double-iteration algorithm, but at low SNR, the estimation accuracy is poor. Performance using adjacent-data symbol to estimate the carrier frequency offset is the worst.

Figure 15 is two users performance comparison of different algorithm, can be seen from the figure, with the increase of the SNR, MSE curves of carrier frequency offset estimation showed a decreasing trend, when the SNR is less than $4 \mathrm{~dB}$, performance of the double-iteration algorithm is better than other algorithms. When the SNR is higher than $4 \mathrm{~dB}$, estimated MSE using interval of five data symbols is very close to double-iteration algorithm. Therefore, the double-iteration algorithm can effectively reduce the impact of noise on the accuracy, frequency offset estimation algorithm based on interval of five data symbols is sensitive to noise and the estimation accuracy is greater affected by noise. Performance using adjacent-data symbol to estimate the carrier frequency offset is the worst.

Figure 16 is four users performance comparison of different algorithm, when the SNR is less than $3 \mathrm{~dB}$, performance of the double-iteration algorithm is better than other algorithms, when the SNR is higher than $3 \mathrm{~dB}$, performance of using interval of five data symbols is better than other algorithms. From this, we can see that in the case of four users interference between users is large, frequency offset estimation algorithm based on interval of five data symbols is sensitive to noise and estimation accuracy is greater affected by noise, the interference between the users have certain robustness, but its performance is very bad at low SNR. Through the comparison and analysis on the performance of each frequency offset estimation algorithm in the case of different number of users, the double-iteration algorithm compared with other algorithms has better performance, estimated MSE curve is very close to the lower bound of estimation. As the double-iteration estimation algorithm using coarse estimation, fine estimation and twice compensation, so as to reduce the effects of noise on performance. 


\section{Conclusion}

In the high-speed mobile environment, LTE-Advanced uplink exist twice the maximum Doppler frequency offset of the carrier frequency offset, resulting in serious ICI and inter-user interference, and bring serious impact to the transmission system. Therefore, the need for carrier frequency offset estimation in receiver. In this paper, for PUCCH format 1, introduce respectively based on pilot symbols, based on the data symbols frequency offset estimation algorithm, and based on the pilot symbols and data joint frequency offset estimation algorithm. On this basis, put forward a frequency offset estimation algorithm suitable for high-speed mobile environment. Through the simulation and performance analysis of variety of methods, the proposed double-iteration algorithm has the best performance, this estimation algorithm using coarse estimation, fine estimation and twice compensation, to reduce the effects of noise on frequency offset estimation and improve estimation accuracy.

\section{Acknowledgements}

This work was supported by Major National Science and Technology Projects (No. 2011ZX03001-003-01) and the Sciences Youth Funded Projects for Chongqing University of Posts and Telecommunications (No.A2012-89). The authors would like to thank the anonymous referees whose insightful comments helped us to improve the presentation of the paper. Meanwhile, express the same thanks to Dr. Li Xiaowen for all his kindness and help.

\section{References}

[1] B. Naik, M B N M, SD Mohiddin Mehata, S D M M, Bojja Durga Bhavani B D B, "GCD Matrix based PAPR Reduction Technique for OFDM System [J]”, International Journal of Signal Processing, Image Processing and Pattern Recognition, vol. 7, no. 5, (2014), pp. 177-186

[2] 3GPP TS 36.211 v11.4, Evolved Universal Terrestrial Radio Access (E-UTRA), Physical channels and modulation, (2013) September.

[3] Z. Shen, A. Papasakellariou, J. Montojo, D Gerstenberger and F. Xu, "Overview of 3GPP LTE-advanced carrier aggregation for $4 \mathrm{G}$ wireless communications", Communications Magazine, (2012) February, vol. 50, no. 2, pp. 122-130.

[4] F T. Chen and C. Wan, "Research and Simulation for Frequency Offset Estimation Algorithm in TDLTE System [J]", Applied Mechanics and Materials, vol. 513, (2014), pp. 1063-1066.

[5] W. Jun, Q. Qin and S. Liu, "Low complexity joint time offset and frequency offset estimations for LTE uplink system [J]", The Journal of China Universities of Posts and Telecommunications, vol. 21, no. 6, (2014), pp. 30-36

[6] I L J. Da Silva, A L F de Almeida and F R P. Cavalcanti, "A new multi-user receiver for PUCCH LTE format 1[C]", Signal Processing Advances in Wireless Communications (SPAWC), 2010 IEEE Eleventh International Workshop on. IEEE, (2010), pp. 1-5.

[7] M. Morelli and M. Moretti, "Carrier frequency offset estimation for OFDM direct-conversion receivers [J]", Wireless Communications, IEEE Transactions on, vol. 11, no. 7, (2012), pp. 2670-2679.

[8] Z. Cvetkovic, V. Tarokh and S. Yoon, "On frequency offset estimation for OFDM [J]", Wireless Communications, IEEE Transactions on, vol. 12, no. 3, (2013), pp. 1062-1072

[9] L. Yang, G. Ren and Z. Qiu, "A novel Doppler frequency offset estimation method for DVB-T system in HST environment [J]", Broadcasting, IEEE Transactions on, vol. 58, no. 1, (2012), pp. 139-143.

[10] E P. Simon, L. Ros and H. Hijazi, "Joint carrier frequency offset and channel estimation for OFDM systems via the EM algorithm in the presence of very high mobility [J]", Signal Processing, IEEE Transactions on, vol. 60, no. 2, (2012), pp. 754-765.

[11] M M U. Gul, S. Lee and X. Ma, "Carrier frequency offset estimation for OFDMA uplink using null subcarriers [J]”, Digital Signal Processing, vol. 29, (2014), pp. 127-137. 


\section{Authors}

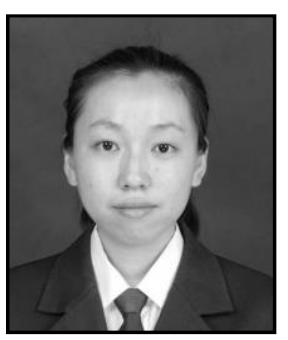

Dan Wang, she was born in China in 1981. She received her $\mathrm{Ph} . \mathrm{D}$. degree in communications and information engineering from Chongqing University, Chongqing, China, in 2014. She received the M.S. degree in circuits and system from Chongqing University, Chongqing, China, in 2006. From 2006 to 2009, she joined Chongqing Chongyou information technology. She has worked on a wide array of wireless communication technologies including TDSCDMA, GSM, and LTE. Since 2009, she has been an engineer in communication engineering, Chongqing University of Posts and Telecommunications and researching on LTE physical layer technology now.

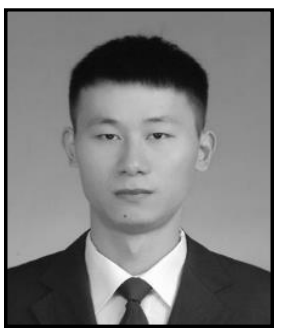

Youbo Feng, he received the B.S. degree from Chongqing University of Posts and Telecommunications, Chongqing, China, in 2013. He is a Postgraduate Student of Chongqing University of Posts and Telecommunications, China, and will receive his M.S. degree in Communication and Information Systems in 2016. His research interests include LTE-Advanced physical layer technology, MIMO communications, and digital signal processing.

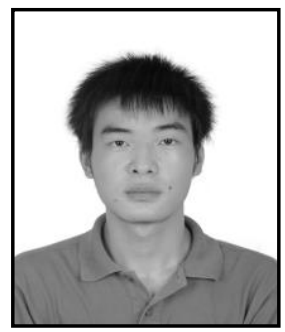

Yiming Lin, he is a Postgraduate Student of Chongqing University of Posts and Telecommunications, China, and will receive his B.S. degree in Communication Engineering in 2016. 